\title{
Derived relations and generalized alteration of preferences
}

\author{
Sonsoles Valdivia-Salas • Michael J. Dougher • \\ Carmen Luciano
}

Published online: 15 December 2012

(C) Psychonomic Society, Inc. 2012

\begin{abstract}
The present study examined the role of derived relations in the generalizability of the evaluative conditioning effect. Healthy university students participated. Four geometrical shapes were first established as discriminative stimuli for the contingent presentation of pictograms (B1, $\mathrm{B} 2, \mathrm{C} 1$, and $\mathrm{C} 2$, respectively). We then assessed the reinforcing properties of $\mathrm{B} 1$ versus $\mathrm{B} 2$, and $\mathrm{C} 1$ versus $\mathrm{C} 2$ by using simultaneous discrimination tasks: at baseline (baseline assessment), after pairing B1 with aversive slides plus noise and B2 with pleasant slides (test I), and after employing equivalence training and testing to establish $\mathrm{B} 1$ as equivalent to $\mathrm{C} 1$ and $\mathrm{B} 2$ as equivalent to $\mathrm{C} 2$ (test II). Most participants $(82 \%)$ in the experimental condition, as compared with the control conditions (17\% and $10 \%$ ), selected the discriminative shapes for B2 (test I) and $\mathrm{C} 2$ (test II) on most trials, replicating and extending previous findings. Subsequently, the geometrical shapes were established as equivalent to the letters $\mathrm{X}, \mathrm{Y}, \mathrm{W}$, and $\mathrm{Z}$, respectively, which then served as antecedent stimuli in simultaneous discrimination tasks as before (test III). As was expected, only participants in the experimental condition showed preference for the novel letters that were established as equivalent to B2-producing and $\mathrm{C} 2$-producing shapes. These findings suggest that the evaluative conditioning effect may extend far beyond the stimulus being de/valuated and narrow the behavioral repertoire.
\end{abstract}

S. Valdivia-Salas $(\square)$

Departamento de Psicología y Sociología, Universidad de

Zaragoza, Campus Ciudad Escolar, Teruel 44003, Spain

e-mail: sonsoval@unizar.es

S. Valdivia-Salas $\cdot$ M. J. Dougher

University of New Mexico, Albuquerque, NM, USA

C. Luciano

University of Almeria, Almeria, Andalusia, Spain
Keywords Transfer of functions · Derived generalization . Evaluative conditioning $\cdot$ Consequence devaluation $\cdot$ Key selection $\cdot$ Humans

Just as the mythical Greek King Midas turned everything he touched into gold, an affectively relevant learning experience may alter a person's previous preferences in diverse life domains. The observed change in liking that is due to the pairing of stimuli has been regarded as an evaluative conditioning (EC) effect (De Houwer, 2007). EC is a form of Pavlovian conditioning in which a neutral stimulus is paired with an affective stimulus and, as a result, the valence of the neutral stimulus changes. The effects have been observed with a number of measures of liking based on Likert scale ratings and reaction time.

Research on EC can be traced back to the early 1950s, but it was the seminal study by Staats and Staats (1957) that stimulated further research on the applications of EC in several domains (for an overview, see De Houwer, Thomas, \& Baeyens, 2001). Staats and Staats showed that participants' responses to a semantic differential scale (pleasantness vs. unpleasantness) were significantly modified after secondorder classical conditioning in which nonsense syllables (CS2) were paired with socially established conditioned stimuli (CS1), such as pretty, healthy, stupid, disgusting (CS2 $\rightarrow$ CS1). Nonsense syllables paired with positive words were evaluated as more pleasant than nonsense syllables paired with negative words.

Subsequent research focused on the possible boundary conditions of EC. Within this line, Tryon and Cicero (1989) explored the generalizability of the EC effect to a third-order by pairing a nonsense syllable (CS2) with a socially established emotive word (CS1), and then a further nonsense syllable (CS3) with CS2 (CS2 $\rightarrow$ CS1 followed by CS3 $\rightarrow$ CS2). Conditioning did occur to the CS2, thus replicating Staats and Staats's (1957) findings, but did not extend to the third-order CS3 (see also Cicero and Tryon, 1989). In a later 
study, Walther (2002, Experiment 4) showed that when nonsense syllables were replaced by more meaningful stimuli, such as pictures of male faces, the EC effect could spread through second-order conditioning procedures. Hammerl and Grabitz (1996) showed that when participants were first exposed to unreinforced paired presentations of two stimuli N1$\mathrm{N} 2$, subsequent EC with N2 altered not only N2 affective valence, but also, and most important, N1 valence (see Walther, 2002, for similar results). The same authors noted that this was the case only when forward (i.e., N1-N2), as opposed to backward (i.e., N2-N1), preconditioning pairing was employed. Overall, these findings demonstrate that, under specific experimental procedures, EC does not require the direct experience of an affectively relevant event to occur. Still, the small number of studies employing higher-order associative paradigms does not allow drawing firm conclusions about the generalizability of the EC effect (Hoffman, De Houwer, Perugini, Baeyens, \& Crombez, 2010).

Within a different approach, Barnes-Holmes, Keane, Barnes-Holmes, and Smeets (2000) showed the generalization of EC to a third-order stimulus by establishing derived stimulus relations through match-to-sample (MTS) procedures. Participants first matched the nonsense syllables VEK and ZID to emotive words CANCER and HOLIDAYS, respectively, and then, the expressions BRAND $X$ and BRAND Y to nonsense syllables VEK and ZID, respectively (summarizing, CANCER $\rightarrow$ VEK; VEK $\rightarrow$ BRAND X; and HOLIDAYS $\rightarrow$ ZID; ZID $\rightarrow$ BRAND Y). Subsequently, participants rated a cola-based drink labeled BRAND Y as significantly more pleasant than an identical drink labeled BRAND X (for a related study, see Smeets \& Barnes-Holmes, 2003). This was the first empirical demonstration of the transfer of EC to a third order, and more generally, of the change in liking via transfer of functions.

Transfer of functions refers to the indirect acquisition of functions by stimuli that participate in classes or relations with other stimuli with established functions. Given its clinical implications, transfer of functions is the most relevant effect associated with derived stimulus relations, or stimulus equivalence. If participants are trained to choose $B$ in the presence of $A$ and $C$ in the presence of $B$, they will most probably show a number of untrained "derived" relations, such as choosing $\mathrm{A}$ in the presence of $\mathrm{B}, \mathrm{B}$ in the presence of $\mathrm{C}, \mathrm{C}$ in the presence of $\mathrm{A}$, and $\mathrm{A}$ in the presence of $\mathrm{C}$. If $\mathrm{A}$ acquires reinforcing functions through pairing with an unconditioned or conditioned reinforcer, and $\mathrm{B}$ and $\mathrm{C}$ acquire similar functions without needing explicit pairing, then we say that $\mathrm{A}, \mathrm{B}$, and $\mathrm{C}$ form an equivalence class (Sidman, 1994) and the reinforcing properties of A have transferred to B and C (Dougher, 1998).

Transfer of functions is now a robust phenomenon in that it has been shown with a number of stimulus functions, including discriminative, respondent, consequential, contextual, and avoidance (for a review, see Dymond \& Rehfeldt, 2000; for more recent evidence, see, e.g., Dougher, Hamilton, Fink, \& Harrington, 2007; Gil, Luciano, Ruiz, \& Valdivia-Salas, 2012; Hooper, Saunders, \& McHugh, 2010; Rodríguez-Valverde, Luciano, \& Barnes-Holmes, 2009). As for the evaluative function, studies by Barnes-Holmes et al. (2000) aforementioned, Dack, Reed, and McHugh (2010; see also Dack, McHugh, \& Reed, 2009), and Smyth, BarnesHolmes, and Forsyth (2007) seem the closest evidence to the derived transfer of the EC effect. With their focus on examining the conditions under which molar versus molecular aspects of various schedules of reinforcement determine EC and its transfer, Dack et al. (2010) showed that participants used nonsense words that had been previously established in an equivalence class with words good and useless to categorize two color circles in whose presence participants were provided with consequences under different schedules of reinforcement. Smyth et al. showed that after pairing a nonsense word with spider-attack scenes, participants reported feeling fearful and disgusted about this nonsense word and, most important, about a different nonsense word that had been previously established in an equivalence relation with the former. However, a direct and fully controlled examination of how derived stimulus relations may contribute, in a sort of domino effect, to a generalized change in liking is still missing.

When direct measures of liking are collected, such as selection responses, the evidence is usually found under the rubric of consequence devaluation. The observation of changes in instrumental responding after devaluation of the reinforcer is a phenomenon extensively demonstrated in the animal literature. In a well-known devaluation study, Colwill and Rescorla (1985) trained rats to make different responses (R1 and $\mathrm{R} 2$ ) to obtain different outcomes (O1 and $\mathrm{O} 2$, respectively). Next, $\mathrm{O} 1$ was devalued via conditioned taste aversion procedures. In the final test, in which the outcomes did not follow selection responses and both R1 and R2 were simultaneously available, R1 was much less frequent than R2. In humans, this effect has been extended by showing that the devaluation of a reinforcer will affect not only the instrumental responses followed by the devalued reinforcer, but also those followed by a nonconditioned stimulus in an equivalence relation with the former (Hayes, Kohlenberg, \& Hayes, 1991; Whelan \& Barnes-Homes, 2004a, b). Hayes et al. first established nonsense symbol B1 as a conditioned reinforcer and nonsense symbol B3 as a conditioned punisher. Subsequently, they used one-to-many MTS procedures to establish $\mathrm{B} 1$ as equivalent to $\mathrm{C} 1$ and $\mathrm{B} 3$ as equivalent to $\mathrm{C} 3$. In the last test, participants' responses to a sorting task were shaped by the contingent presentation of nonconditioned $\mathrm{C} 1$ and $\mathrm{C} 3$; that is, the probability of responses followed by $\mathrm{C} 1$ (in an equivalence relation with conditioned reinforcer B1) was higher than those followed by $\mathrm{C} 3$ (in an equivalence relation 
with conditioned punisher B3). Whelan and Barnes Holmes (2004b, Experiment 4) showed similar results, but using relations other than equivalence. The authors first established visual symbol $\mathrm{X} 1$ as a conditioned reinforcer and $\mathrm{B} 2$ as a conditioned punisher and then used MTS procedures to establish B2 as equivalent to C2 and the opposite of C1. During the test, selection responses to three nonsense syllables (NSS) were followed by $\mathrm{C} 1$ (i.e., derived reinforcer), $\mathrm{C} 2$ (i.e., derived punisher), or a novel visual symbol, respectively. All 4 participants eventually chose the NSS followed by $\mathrm{C} 1$ on most trials (for a related study using other stimulus relations, see Whelan, Barnes-Holmes, \& Dymond, 2006). Overall, these findings suggest that, in the case of humans, the impact of reinforcer devaluation on behavior may go beyond the actual contingency being altered and extend to nonconditioned stimuli.

The present research aims to extend previous findings, first, by isolating and, second, by further exploring the role of derived stimulus relations in the generalizability of EC. One of the main concerns regarding the aforementioned studies has to do with the test conditions. In Hayes et al. (1991), tests included the contingent presentation of the derived consequences across a large number of trials. As the authors noted, the extensive exposure to testing trials might have shaped the intended performance, regardless of the function supposedly acquired by the consequences of responding. In Whelan and Barnes-Holmes (2004a, 2004b), conditioning trials were intermixed with test trials during testing blocks, which might lead to the conclusion that reconditioning is necessary for the effect of derived relations on change in preferences to occur and maintain across trials. Aiming at isolating the impact of derived relations on consequence value alteration, in the first part of the study, we implemented a variation of the former procedures, mainly affecting the test conditions. As compared with those procedures, (1) none of the responses during the tests were followed by the contingent presentation of the consequences, (2) tests trials were not intermixed with conditioning trials, and (3) tests were considerably shortened. Briefly, following baseline assessment of preferences for arbitrary stimuli B1, B2, C1, and $\mathrm{C} 2$, we paired $\mathrm{B} 1$ with unpleasant stimuli and $\mathrm{B} 2$ with pleasant stimuli (i.e., EC with B1 and B2) and then trained stimulus relations so that $\mathrm{B} 1$ was in an equivalence relation with $\mathrm{C} 1$ and $\mathrm{B} 2$ was in an equivalence relation with $\mathrm{C} 2$ (i.e., equivalence training and testing). Selection responses to Bproducing and $\mathrm{C}$-producing shapes were reassessed after implementing each intervention. Two control conditions were added to further isolate the effect of un/pleasant pairings and stimulus equivalence procedures on participants' changes in preferences for conditioned B and nonconditioned C stimuli.

In the second part of the study, we explored the extent of the generalizability of EC by relating B-producing and Cproducing shapes to novel stimuli (i.e., letters $\mathrm{X}, \mathrm{Y}, \mathrm{W}$, and
Z). These stimuli then served as antecedent stimuli in novel preference tasks in which, as before, none of the participants' responses was followed by the contingent presentation of the consequence. If experimental participants, as compared with control participants, selected the letters that had been related to $\mathrm{B} 2$-producing and $\mathrm{C} 2$-producing shapes, we would have provided a laboratory model of the derived generalization of $\mathrm{EC}$ effect across novel and otherwise unrelated stimuli events.

\section{Method}

\section{Participants}

University students were recruited through online announcements and personal contact. A total of 59 students met the inclusion criteria and participated in the study (23 men, 1930 years old), 46 at the University of New Mexico (UNM) and 13 at the University of Almeria (UAL). Students at UNM received academic credits for their participation. Students at UAL received a ticket for a free breakfast in the university cafe.

Before beginning the experiment proper, the experimenter described the general procedure and participants read and signed a statement of informed consent, which included a statement explicitly informing them that they could discontinue participation at any time without giving up their credits/tickets. Upon completion of the study, participants were fully debriefed.

\section{Setting and materials}

At both UNM and UAL, the experiment was run in a laboratory room equipped with a table, a personal computer with a keyboard and 14-in. color monitor, two chairs, an armchair, a floor lamp, and file organizers. Participants sat at a table in front of the personal computer. All stimuli were presented on the monitor. The computer ran a customized Visual Basic program (available upon request to the authors) that controlled the experimental tasks and recorded participants' responses. Some tasks required participants to use the keyboard to select visual stimuli that appeared on the screen. Visual stimuli included four geometric shapes (square, circle, hexagon, and triangle) and the six pictograms shown in Fig. 1 (columns B and C; column A includes the nonsense syllables used during equivalence training and testing). These were displayed in black on a gray background. In addition, pleasant and unpleasant slides were presented on conditioning trials during stimulus pairing procedures. Unpleasant slides were selected from the International Affective Picture System (Center for the Study of Emotion and Attention, CSEA-NIMH, 2001) and depicted injured and/or disfigured bodies, rated low for pleasure, high for arousal, and low for dominance according to normative criteria (Lang, Bradley, \& Cuthbert, 2001). The 
Fig. 1 Arbitrary visual stimuli utilized during the procedure

\begin{tabular}{|c|c|c|c|}
\hline & A & B & $\mathrm{C}$ \\
\hline 1 & $\mathrm{MIF}$ & & \\
\hline 2 & NAZ & & \\
\hline 3 & VEK & & \\
\hline
\end{tabular}

specific numbers of the slides used were 3053, 3063, 3069, $3102,3261,3170,3080,3000,3168$, and 3062. In addition to the slides, on aversive conditioning trials, a 100-dBA white-noise burst played through headphones was also presented. Noise stimuli of this intensity are aversive but not physiologically harmful (Hobbs, 1990). Pleasant slides were extracted from various Web sites and selected from a pool of 20 images depicting babies and/or pets in funny situations on the basis of six independent observers' ratings. No auditory stimuli were presented with the pleasant slides.

\section{Variables and experimental design}

We employed a pre-post design with both within-subjects and between-conditions comparisons of the effects of three experimental manipulations on the alteration of the relative preference for a number of stimuli.

As is depicted in Fig. 2, participants were randomly assigned to one of three conditions during the first part of the experiment. In the conditioning and classes condition (CondClass), participants were exposed to differential EC procedures in which B1 was followed by unpleasant slides and noise and B2 was followed by pleasant slides. Thereafter, match-to-sample (MTS) training and testing procedures were implemented to establish two 3-member equivalence classes (A1-B1-C1; $\mathrm{A} 2-\mathrm{B} 2-\mathrm{C} 2)$. In the conditioning and no-classes condition (CondNoClass), participants were exposed to differential EC procedures but were not exposed to the equivalence training and testing. In the no-treatment condition (NoTreat), participants were exposed to a stimulus pairing procedure in which both the unpleasant slides with the noise and the pleasant slides were preceded by an asterisk (instead of B1 and B2), and they were not exposed to equivalence training and testing. Simultaneous discrimination tasks in which geometrical shapes served as antecedent stimuli were presented at baseline, after differential EC phase (test I), and after equivalence training and testing phase (test II). After screening procedures, 34 participants were assigned to CondClass, 15 to CondNoClass, and 10 to NoTreat (see the Data Analysis of Changes in Preference section for the reasons why the number of participants was so unequal across conditions).
In the second portion of the study, participants in all three conditions underwent a visual pairing procedure in which the shapes that served as antecedent stimuli during the simultaneous discrimination task at baseline, test I, and test II (i.e., geometrical shapes: square, circle, hexagon, and triangle) were equated to novel stimuli (i.e., letters $\mathrm{X}, \mathrm{Y}, \mathrm{W}$, and $\mathrm{Z}$, respectively). For the ease of reading, we will refer to this procedure with the colloquial term domino effect intervention from now on. Subsequently, participants were presented with simultaneous discrimination tasks in which the letters, instead of the shapes, served as antecedent stimuli (test III).

\section{Procedure}

After signing the consent form, participants were administered the Beck Depression Inventory (BDI-II; Beck, Brown, \& Steer, 1996) and the Post-Traumatic Stress Disorder (PTSD) Checklist for civilian population (PCL-C; Smith et al., 1997). Anyone scoring 13 or more on the BDI-II, and/or meeting two of the three criteria for a diagnosis of PTSD on the PCL-C was dismissed from the study in order to minimize unintended effects of the aversive stimuli during EC.

The experimental procedures consisted of eight phases (see Fig. 2), some of which had both training and test components. All phases were conducted in a single session that lasted about $1 \mathrm{~h}$. The specific instructions for each phase were read aloud by the experimenter, who stayed in the experimental room and sat behind the participant during each training component but left the room during each of the testing components.

\section{Phase 1: Operant training and baseline assessment}

The purpose of this phase was, first, to train four pictograms as consequences for selection responses to the geometrical shapes (square, circle, hexagon, and triangle, respectively) and, second, to test for the baseline reinforcing properties of the pictograms, measured by the number of selection responses to the geometrical shapes. Participants were presented with two simultaneous discrimination tasks (square vs. circle and hexagon vs. triangle), each consisting of 27 trials: the first 12 trials for the training, and the last 15 for the testing part of the phase. 
Fig. 2 Schematic overview of the procedures employed in each condition

\begin{tabular}{|c|c|c|}
\hline CondClass Cond. & CondNoClass Cond. & NoTreat Cond. \\
\hline \multicolumn{3}{|c|}{$\begin{array}{c}\text { PHASE 1 } \\
\text { Operant training } \\
(8 \text { trials per pair) } \\
\text { Square vs. Circle (followed by B1 and B2, respectively) } \\
\text { Hexagon vs. Triangle (followed by C1 and C2, respectively) }\end{array}$} \\
\hline \multicolumn{3}{|c|}{$\begin{array}{c}\text { Baseline assessment } \\
\text { (15 trials per pair) } \\
\text { Square vs. Circle (no consequences) } \\
\text { Hexagon vs. Triangle (no consequences) }\end{array}$} \\
\hline \multicolumn{3}{|c|}{ PHASE 2} \\
\hline $\begin{array}{r}\text { Evaluative Condition } \\
\text { B1 } \rightarrow \text { unpleas } \\
\text { B } 2 \rightarrow \text { pleasan }\end{array}$ & $\begin{array}{l}\text { g with B stimuli } \\
\text { it slide plus noise } \\
\text { lide }\end{array}$ & $\begin{array}{l}\text { Presentation of slides } \\
* \rightarrow \text { unpleasant slide plus noise } \\
* \rightarrow \text { pleasant slide }\end{array}$ \\
\hline \multicolumn{3}{|c|}{$\begin{array}{c}\text { PHASE 3 } \\
\text { Test I } \\
\text { Square vs. Circle (no consequences) } \\
\text { Hexagon vs. Triangle (no consequences) }\end{array}$} \\
\hline \multicolumn{3}{|l|}{$\begin{array}{c}\text { Equiv Training and Testing } \\
\text { A1-B1-C1 } \\
\text { A2-B2-C2 }\end{array}$} \\
\hline \multicolumn{2}{|c|}{$\begin{array}{l}\text { Re-Conditioning } \\
\mathrm{B} 1 \rightarrow \text { unpleasant slide plus noise } \\
\mathrm{B} 2 \rightarrow \text { pleasant aversive slide }\end{array}$} & $\begin{array}{l}\text { Presentation of slides } \\
* \rightarrow \text { unpleasant slide plus noise } \\
* \rightarrow \text { pleasant slide }\end{array}$ \\
\hline \multicolumn{3}{|c|}{$\begin{array}{c}\text { PHASE 6 } \\
\text { Test II } \\
\text { Square vs. Circle (no consequences) } \\
\text { Hexagon vs. Triangle (no consequences) }\end{array}$} \\
\hline \multicolumn{3}{|c|}{$\begin{array}{c}\text { PHASE } 7 \\
\text { Domino effect intervention } \\
\mathrm{X}=\square \\
\mathrm{Y}=\square \\
\mathrm{W}=\square \\
\mathrm{Z}=\triangle\end{array}$} \\
\hline & $\begin{array}{r}\text { PHASE 8 } \\
\text { Test III } \\
\text { X vs. Y (no cons } \\
\text { W vs. Z (no cons }\end{array}$ & \\
\hline
\end{tabular}

To start, four forced choice trials were presented: one shape (either square or circle) was displayed either on the left or on the right side of the screen. When the corresponding key was pressed, the shape disappeared, and the associated pictogram was flashed at the top center of the screen for $2 \mathrm{~s}$. There were two forced choice trials with both the square and the circle, presented in a fixed sequence across participants.

A series of eight simultaneous discrimination trials followed. Here, the two shapes (square and circle) were presented simultaneously, their location on the screen (left vs. right) counterbalanced across trials. Participants selected one of the shapes by pressing 1 or 8 on the keyboard. Every selection of the square was followed by the presentation of pictogram 1, and every selection of the circle was followed by pictogram 2 .
After the eighth trial, the experimenter administered a quick pencil-and-paper test to assess participants' acquisition of the relations between the shapes (square and circle) and their respective pictograms. All responded correctly and continued participation. The experimenter then advised participants that the pictograms would not be shown anymore after pressing 1 or 8 . Instead, the computer would record their choices and, at the end of the whole experiment, present them with what was related to the shapes they chose as many times as they chose them. Thus, participants were told to pick the shapes according to what they wanted to be presented with at the end of the experiment.

A total of 15 simultaneous discrimination trials, identical to those described above, were then presented. The only 
difference was that none of the trials was followed by the corresponding pictogram. Instead, the screen turned blank for $3.5 \mathrm{~s}$. The number of responses to each shape on these last 15 trials determined the baseline reinforcing properties of pictograms 1 and 2 .

Subsequent to this, baseline reinforcing properties of pictograms 3 and 4 were assessed in the same way as for pictograms 1 and 2, except that instead of a square and a circle, a hexagon and a triangle were used as discriminative stimuli for pictograms 3 and 4, respectively. Once the reinforcing properties of the four pictograms were established, the computer automatically assigned, for each participant, an alphanumerical label to each pictogram for use in the subsequent $\mathrm{EC}$ and stimulus equivalence phases of the experiment. Of pictograms 1 and 2, the most reinforcing pictogram was labeled B1, and the other B2. Of pictograms 3 and 4 , the most reinforcing pictogram was labeled $\mathrm{C} 1$, and the other $\mathrm{C} 2$. Thus, for each participant, $\mathrm{B} 1$ and $\mathrm{C} 1$ were always more reinforcing than $\mathrm{B} 2$ and $\mathrm{C} 2$, respectively, regardless of the actual pictograms that were so designated. Figure 1 depicts a particular assignment of alphanumeric labels that, depending on their assessed reinforcing properties, may not have been true for all participants.

\section{Phase 2: Evaluative conditioning with B1 and B2}

By means of a delayed conditioning procedure, the pictogram labeled B1 was paired with the unpleasant slides along with the $100 \mathrm{dBA}$ white-noise burst. The pictogram labeled B2 was paired with the pleasant slides.

Conditioning trials with $\mathrm{B} 1$ and $\mathrm{B} 2$ were interspersed in a 24-trial series containing 8 conditioning trials and 4 extinction trials per B stimulus. On conditioning trials, either B1 or B2 was presented centered at the top of the screen. After $6 \mathrm{~s}$, either an unpleasant slide plus noise or a pleasant slide was presented at the center of the screen just below B1 or B2 for $4 \mathrm{~s}$. After that, the screen cleared for a 6-s intertrial interval (ITI) before the next trial was presented. Slides varied randomly without replacement across trials. On extinction trials, B1 or B2 was displayed at the top of the screen for $6 \mathrm{~s}$ and then disappeared. A 10-s ITI preceded the next trial. Extinction trials were used in an attempt to avoid rapid extinction in subsequent phases of the experiment where the pictograms were not always followed by slides (Catania, 1998). For participants in NoTreat, where conditioning with the B stimuli was absent, the series was identical, except that an asterisk replaced the B stimuli. The asterisk was presented to warn participants of the impending slide and focus their attention on the screen.

\section{Phase 3: Test I}

To start this phase, the experimenter presented the same pencil-and-paper tests as those used in phase 1 to test for participants' retention of the previously trained relations between geometrical shapes and pictograms. Again, all participants responded correctly. Then a series of 30 simultaneous discrimination trials was presented, preceded by the same instructions as at the baseline assessment of phase 1 . The first 15 trials assessed the reinforcing properties of B1 versus B2 as the number of responses to square versus circle, and the last 15 assessed reinforcing properties of $\mathrm{C} 1$ versus $\mathrm{C} 2$ as the number of responses to hexagon versus triangle. As in the baseline assessment, two geometrical shapes were simultaneously flashed on the screen (either square and circle or hexagon and triangle), and participants responded by using the keyboard. Note that, as during baseline assessment, none of the participants' responses was followed by presentation of the associated pictograms.

\section{Phase 4: Equivalence training and testing}

A one-to-many MTS procedure was used to train and test the formation of two 3-member equivalence classes (A1-B1$\mathrm{C} 1 / \mathrm{A} 2-\mathrm{B} 2-\mathrm{C} 2)$. Alphanumerical labels are used here for descriptive purposes only; they were not presented to the participants. As is depicted in Fig. 1, the A stimuli, which served as samples during training, were nonsense syllables. The B and C stimuli, which served as comparisons during training, were abstract symbols and black-and-white-line patterns, respectively. The relations $\mathrm{A} 1-\mathrm{B} 1, \mathrm{~A} 1-\mathrm{C} 1, \mathrm{~A} 2-\mathrm{B} 2$, and $\mathrm{A} 2-\mathrm{C} 2$ were trained in blocks of four trials (one per relation). On each trial, the sample was presented at the top center of the screen, and after a 1-s delay, three comparisons appeared across the bottom. No response was required to the sample stimulus. Comparisons were always from the same alpha group (e.g., B1, B2, B3). B3 and C3 served as incorrect comparisons and were used to control for responding by exclusion (see Carrigan \& Sidman, 1992). The spatial location of the comparisons varied randomly across trials. Participants selected from among the comparisons using the keyboard. Pressing key 1 selected the left comparison, key 5 selected the comparison in the middle, and key 8 selected the comparison on the right. Correct responses were followed by the written message CORRECT for $1.5 \mathrm{~s}$; incorrect responses were followed by the written message WRONG. The training criterion was set at $100 \%$ correct performance over eight consecutive 4-trial blocks (32 trials). When achieved, participants were presented with symmetry and equivalence test trials in extinction (i.e., no feedback). Symmetrical relations (B1$\mathrm{A} 1, \mathrm{C} 1-\mathrm{A} 1, \mathrm{~B} 2-\mathrm{A} 2$, and $\mathrm{C} 2-\mathrm{A} 2$ ) were tested first in blocks of 4 trials (1 per relation). The test criterion was set at $100 \%$ correct performance over three correct 4-trial blocks (12 trials). Equivalence relations (B1-C1, C1-B1, B2-C2, and C2-B2) were then tested in the same way with the same criterion. If participants did not meet the criteria, they were reexposed to the training procedure, and the tests were repeated. Participants not 
meeting the test criteria after their third exposure to the testing blocks were dropped from further participation.

\section{Phase 5: Reconditioning with B1 and B2}

Procedures identical to those in phase 2 were used to recondition B1 and B2, except that three novel slides were selected for each pictogram. IAPS slides 3168,3062 , and 3150 were used with B1. A series of six trials (three per stimuli) was presented randomly without replacement.

\section{Phase 6: Test II}

The pencil-and-paper test was again administered to test for the retention of the previously trained relations. All participants passed the test. Afterward, a series of 30 simultaneous discrimination trials identical to that in phase 3 was presented.

\section{Phase 7: Domino effect intervention}

To start this phase, the experimenter presented the participants with a sheet of paper (see Fig. 2) that equated each of four letters (X, Y, W, and Z) with one of the shapes (square, circle, hexagon, and triangle) used as antecedent stimuli in previous simultaneous discrimination tasks (i.e., phases 1, 3, and 6).

\section{Phase 8: Test III}

A series of 30 simultaneous discrimination trials was then presented. On the first 15 trials, participants chose between $X$ and $\mathrm{Y}$, and on the last 15 trials, they chose between $\mathrm{W}$ and $\mathrm{Z}$. As on previous tests, none of the trials was followed by the presentation of any stimuli. Due to technical problems with the software, 3 participants assigned to CondClass did not go through this test.

Upon completion of these trials, participants were informed that they would not be exposed to the announced final phase. After debriefing, they left.

\section{Data analysis of changes in preference}

We first conducted analyses including all the participants in order to examine the impact of each of the three interventions on the average number of selection responses to B2- and C2producing stimuli across tests and depending on the condition to which participants were assigned (the results of this analysis are shown in Table 1). Specifically, the average number of selection responses was subjected to a 2 (stimulus: B2producing, C2-producing) $\times 4$ (phase: baseline, test I, test II, test III) $\times 3$ (condition: CondClass, CondNoClass, NoTreat) mixed-design ANOVA, with condition as a between-subjects factor. If the higher-order interaction was significant, separate 2 (stimulus) $\times 3$ (condition) mixed ANOVAs were conducted on each test phase. Post hoc tests using the Bonferroni correction allowed us to examine the differences between conditions in the average number of selection responses within each test. When necessary, the Greenhouse-Geisser degrees of freedom adjustment procedure was applied to the repeated measures factors to correct for violations of sphericity common to ANOVAs. Partial eta squared $\left(\eta_{\mathrm{p}}{ }^{2}\right)$ was calculated in order to determine the effect size of preference alteration. Following Cohen's (1988) guidelines, .01, .10, and .25 were used as thresholds to define small, medium, and large effects, respectively.

Considering that our critical test was test III, we conducted a second analysis of the data, including only those participants who, during test I and test II, showed the expected alteration of preferences. Here, we did not examine the average preference on each condition but the percentage of participants in each condition who met what we called a preference shift criterion. For present purposes, a preference shift was defined as a change of at least six selection responses across relevant test conditions. Thus, to say that the conditioning procedures in phase 2 produced a preference shift from B1- to B2-producing shape, participants had to emit six fewer B1 responses and, necessarily, six more B2 responses on the 15 assessment trials in test I, relative to the 15 assessment trials in baseline; the same was true for the remaining tests. The application of this criterion reduced the number of participants whose performances were analyzed through the final test from 34 to 11 in CondClass, and from 15 to 12 in CondNoClass. The performance of the 10 participants initially assigned to NoTreat condition was analyzed through test III (see Table 2 for details on the actual number of participants in each condition who met criteria across tests). Chi-square analyses were conducted to test for differences between conditions in the percentage of participants showing a preference shift in each test phase.

\section{Results}

All participants in the CondClass condition met the test criteria for the establishment of the equivalence classes within two cycles of testing.

The effect of the interventions on the average change in preferences in each condition across phases

Table 1 shows the mean and standard deviation of selection responses to $\mathrm{B} 2$-producing and $\mathrm{C} 2$-producing stimuli across tests on each condition. The 2 (stimulus) $\times 4$ (phase) $\times 3$ (condition) mixed ANOVA revealed several effects - chief among them, the significant interaction between stimulus type, phase, and condition, $F(5.1,128.7)=3.7, p=.003$, $\eta_{\mathrm{p}}{ }^{2}=.129$, suggesting that, on average, the number of B2- 
Table 1 Mean and standard deviation of selection responses to B2-producing and C2-producing stimuli across test phases in each condition

Test/Condition Stimulus

\begin{tabular}{cccc}
\hline B2-producing & & \multicolumn{2}{c}{ C2-producing } \\
\hline$M$ & $S D$ & $M$ & $S D$
\end{tabular}

\section{Baseline}

CondClass

CondNoClass

NoTreat

Test I

CondClass

CondNoClass

NoTreat

\section{Test II}

3.3

4.5

4.0
2.8

2.8

3.5
2.8

4.5

4.3
2.8

2.6

3.3

$\begin{array}{lrrrr}\text { CondClass } & 13.8^{\mathrm{a}} & 2.5 & 11.6^{\mathrm{a}} & 5.2 \\ \text { CondNoClass } & 14.1^{\mathrm{a}} & 2.5 & 7.2^{\mathrm{b}} & 4.5 \\ \text { NoTreat } & 5.2^{\mathrm{b}} & 4.1 & 4.7^{\mathrm{b}} & 3.9\end{array}$

\section{Test III}

\begin{tabular}{lrrrr} 
CondClass* & $14.1^{\mathrm{a}}$ & 2.3 & $12.1^{\mathrm{a}}$ & 4.8 \\
CondNoClass & $14.5^{\mathrm{a}}$ & 1.8 & $7.7^{\mathrm{b}}$ & 5.1 \\
NoTreat & $5.1^{\mathrm{b}}$ & 4.0 & $6.4^{\mathrm{b}}$ & 4.4 \\
\hline
\end{tabular}

Note. Gray shadowing indicates the stimulus that the intervention prior to each test (evaluative conditioning with B1 and B2, equivalence training and testing, and domino effect intervention, respectively) was directed to. CondClass, conditioning and classes condition $(N=34)$; CondNoClass, conditioning and no-classes condition $(N=15)$; NoTreat, no-treatment condition $(N=10)$. Means with different superscripts differed $(p<.03)$ for within-phase comparisons between experimental conditions.

$* N=28$.

producing and $\mathrm{C} 2$-producing responses varied across tests depending on whether participants were assigned to the CondClass, CondNoClass, or NoTreat condition. We also found another interaction that could be subsumed under the significant three-way interaction: stimulus type $\times$ phase, $F(2.5$, $129)=11.16, p<.001, \eta_{\mathrm{p}}{ }^{2}=.18$. In order to untangle the threeway interaction, separate 2 (stimulus) $\times 3$ (condition) ANOVAs were conducted on each test phase. During baseline, average selection responses to $\mathrm{B} 2$-producing and $\mathrm{C} 2$-producing

Table 2 Number (percentage) of participants per condition who showed a shift in preferences (Yes) during test I, test II, and test III from the total number of participants whose performance was analyzed stimuli did not discriminate between conditions $(p s>.30)$. During test I, we found a significant interaction between stimulus and condition, suggesting that selection responses to B2producing and $\mathrm{C} 2$-producing stimuli varied as a function of the condition to which participants were assigned. Post hoc analyses revealed that the number of selection responses to B2producing shape in CondClass $(M=13.2)$ and CondNoClass $(M=13.0)$ conditions differed significantly from that in the NoTreat condition $(M=5.2), p<.001$. As for the selection of

in each test (Tot); the number of participants who shifted their preference from $\mathrm{C} 1$ to $\mathrm{C} 2$ after conditioning with the $\mathrm{B}$ stimuli is also indicated (Generalized), but it is not considered in subsequent tests

\begin{tabular}{|c|c|c|c|c|c|c|}
\hline & \multicolumn{2}{|l|}{ CondClass } & \multicolumn{2}{|l|}{ CondNoClass } & \multicolumn{2}{|c|}{ NoTreat } \\
\hline & Yes + Generalized & Tot & Yes + Generalized & Tot & Yes & Tot \\
\hline Test I & $17+11(82)$ & 34 & $12+1(86.7)$ & 15 & $1(10)$ & 10 \\
\hline Test II & $14(82)$ & 17 & $2(17)$ & 12 & $1(10)$ & 10 \\
\hline Test III & $11(100)^{*}$ & 11 & $2(17)$ & 12 & $1(10)$ & 10 \\
\hline
\end{tabular}

*Of the 14 participants who shifted their preference during test II, only 11 went through test III. 
C2-producing shape, we found a trend toward a significant difference between CondClass $(M=8.2)$ and NoTreat $(M=4.1)$ conditions, $p<.06$. Still, a paired sample $t$-test revealed that, in the CondClass condition, but not the NoTreat condition, the average number of selection responses to the B2producing shape differed significantly from the average to the C2-producing shape, $t(33)=5.25, p<.001$. Taken together, the pattern of selection responses to B2- and C2-producing shapes during Test I suggests that, as was expected, EC with B1 and B2 altered preferences for the B2-producing shape more reliably than for the C2-producing shape.

The ANOVA during test II also revealed a significant interaction between stimulus and condition, $F(2,56)=8.36$, $p=.001, \eta_{\mathrm{p}}{ }^{2}=.23$. As was expected, the three conditions showed the same pattern of selection responses to the B2producing shape as during test $\mathrm{I}$, with CondClass $(M=13.8)$ and CondNoClass $(M=14.1)$ differing significantly from NoTreat $(M=5.2), p s<.001$. After establishing a derived relation of equivalence between B2 (conditioned appetitive stimulus) and C2 in CondClass only, the average number of selection responses to the $\mathrm{C} 2$-producing shape was significantly larger for CondClass $(M=11.6)$ than for CondNoClass $(M=7.2)$ and NoTreat $(M=4.7), p s<.03$.

During test III, selection of B2-producing and C2producing letters varied by condition, as supported by the significant stimulus $\times$ condition interaction, $F(2,50)=10.44$, $p<.001, \eta_{\mathrm{p}}{ }^{2}=.29$. As during test II, the average number of selection responses to the B2-producing letter in CondClass $(M=14.1)$ and CondNoClass $(M=14.5)$ differed significantly from that in NoTreat $(M=5.1), p s<.001$, and the average number of selection responses to the $\mathrm{C} 2$-producing letter in CondClass $(M=12.1)$ varied significantly from that in CondNoClass $(M=7.7)$ and NoTreat $(M=6.4), p \mathrm{~s}<.02$.

The effect of the interventions on the percentage of participants in each condition who met the preference shift criterion

The individual analysis of the data yielded similar results. Table 2 indicates the number and percentage of participants per condition who shifted their preferences from B1- and C1- to B2- and C2-producing stimuli on each test. Most participants in CondClass $(82 \%)$ and CondNoClass $(86.7 \%)$ conditions demonstrated a shift in their preferences from B1- to B2-producing shape in test I, relative to baseline, but not from $\mathrm{C} 1$ to $\mathrm{C} 2$. Only 1 NoTreat participant (10\%) altered his preferences from B1- to B2-producing shape. A closer look at the data reveals that $30 \%$ (11 of the 28 CondClass and 1 of the 13 CondNoClass) of the total participants who shifted their preferences from B1- to B2producing shape also showed a shift in preference from $\mathrm{C} 1$ to $\mathrm{C} 2$-producing shape, although no derived relation between $\mathrm{B}$ and $\mathrm{C}$ stimuli had yet been established. We will comment on this finding in the Discussion section. As compared with CondNoClass $(17 \%)$ and NoTreat $(10 \%)$ participants, most CondClass participants $(82 \%)$ shifted their preference from $\mathrm{C} 1$ - to $\mathrm{C} 2$-producing shape in test II, as would be expected due to the equivalence relation established between B1 and $\mathrm{C} 1$ and between B2 and C2 immediately prior to this test. Similar results were obtained in test III. That is, all CondClass participants whose performance was analyzed through this last test chose the letters that had been established as B2-producing and C2-producing in the previous phase. Only $17 \%$ of CondNoClass and $10 \%$ of NoTreat participants showed this pattern.

In statistical terms, a shift in preferences from B1- to B2producing shape during test I was significantly more likely to occur in CondClass and CondNoClass than in NoTreat participants (83.67\% vs. $10.00 \%$, respectively, $p<.001$, Fisher's exact test). Likewise, a shift in preferences from $\mathrm{C} 1$ - to $\mathrm{C} 2$-producing shape during test II was more likely to occur in CondClass than in CondNoClass and NoTreat participants $\left(82.35 \%\right.$ vs. $13.64 \%$, respectively, $\chi^{2}(1)=$ $18.42, p<.001)$. Chi-square analyses also revealed a significant relationship between participants' pattern of selection responses during test II and test III, $\chi^{2}(1)=25.32, p<$ .001. For instance, participants who shifted preferences from $\mathrm{B} 1$ and $\mathrm{C} 1$ to $\mathrm{B} 2$ and $\mathrm{C} 2$ during test II (i.e., in CondClass) selected the letters related to B2-producing and C2-producing shapes during test III, and participants who showed preferences for B2 and $\mathrm{C} 1$ during test II (i.e., CondNoClass) selected the letters related to B2-producing and C1-producing shapes during test III.

\section{Discussion}

The purpose of the present research was to explore the impact of derived relations on the generalizability of EC. In the first part of the study, we employed a variation of the procedures employed by Whelan and Barnes-Holmes (2004b) to isolate the impact of relational training and testing on the transformation of the consequential function of events (in our study, $\mathrm{C} 1$ and $\mathrm{C} 2$ ). In the second part of the study, we established relations between the antecedent stimuli used in baseline, test I, and test II (i.e., geometrical shapes) and the letters X, Y, W, and $Z$. These letters then served as antecedent stimuli in simultaneous discrimination tasks.

Figure 3 depicts the cascade of events that followed differential EC with B1 and B2. The first thing we observed is the change in preference from B1-producing to B2producing shape in test $\mathrm{I}$, relative to baseline, only for participants who underwent conditioning with B stimuli (CondClass and CondNoClass conditions). That is, upon pairing otherwise neutral stimuli with pleasant (humorous slides) and unpleasant (mutilation slides plus noise) stimuli, 
Fig. 3 Depiction and description of the relations established during the procedure, both directly and by derived means, and different levels of impact resulting from evaluative conditioning with B1 and B2

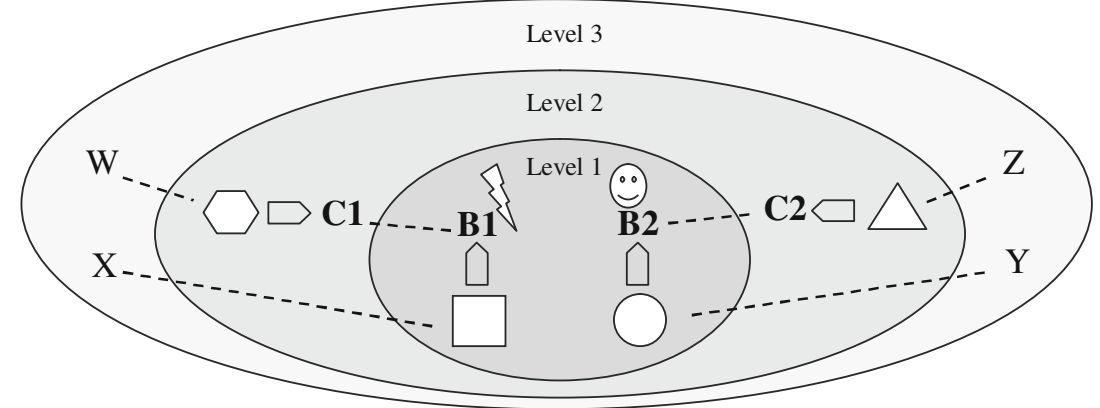

Note: Symbol $\square$ depicts discriminated operants. Dotted lines depict relations in which relational processing was involved.

Level 1: evaluative conditioning with B1 and B2 alters the relative evocative function of square and circle, discriminative for conditioned punisher B1 and conditioned reinforcer B2, respectively: most participants in CondClass and CondNoClass, as compared to NoTreat, selected circle.

Level 2: the equivalence between $\mathrm{B} 1$ and $\mathrm{C} 1$ and between $\mathrm{B} 2$ and $\mathrm{C} 2$ alters the relative evocative function of hexagon and triangle, discriminative for nonconditioned $\mathrm{C} 1$ and $\mathrm{C} 2$, respectively: most participants in CondClass, as compared to CondNoClass and NoTreat, selected triangle.

Level 3: the equivalence between square, circle, hexagon and triangle, and novel stimuli $\mathrm{X}, \mathrm{Y}, \mathrm{W}$, and $\mathrm{Z}$, respectively, establishes $\mathrm{Y}$ and $\mathrm{Z}$ as $\mathrm{S}^{\mathrm{d}}$-like stimuli, and $\mathrm{X}$ and $\mathrm{W}$ as $\mathrm{S}^{\Delta}$-like stimuli: most participants in CondClass, as compared to CondNoClass and NoTreat, selected Y and Z.

most participants selected the shape that signaled the availability of the positive conditioned stimulus. Although we used direct measures of preference (i.e., selection responses) instead of self-reported measures of liking, these findings resemble those of the EC effect. Most important, the experimental preparation provides both within- and betweenparticipants comparisons of the effect of EC procedures, as recommended by De Houwer et al. (2001). We note that, given that the rate of reinforcement was the same for both response options (remember that during the tests, none of the response options was followed by any stimulus presentation), the only plausible explanation to the observed change in preference during test $I$ is the alteration of reinforcement value.

As was noted in the Results section, $30 \%$ of the total participants who shifted their preferences from B1producing to B2-producing shape during test I also showed an unexpected shift in preference from $\mathrm{C} 1$-producing to $\mathrm{C} 2$ producing shape, although no experimental relation had yet been established between $\mathrm{B} 1$ and $\mathrm{C} 1$ and between $\mathrm{B} 2$ and $\mathrm{C} 2$. A closer look at the performance of these participants reveals that most of them showed strong preferences for B1producing and $\mathrm{C} 1$-producing shapes at the phase 1 baseline assessment. The average ratios of B1-producing to B2producing and $\mathrm{C} 1$-producing to $\mathrm{C} 2$-producing responses among this group were $12 / 3$ (range of B1-producing responses $=8-15)$ and $13 / 2($ range $=8-15)$, as compared with average ratios of $11 / 4$ (range $=8-15)$ and $10 / 5$ (range $=$ $8-15)$, respectively, for the other 29 participants in these groups. The preference shift from $\mathrm{C} 1$-producing to $\mathrm{C} 2$ producing shape after conditioning with just the $\mathrm{B}$ stimuli for these participants implies that stimulus preference defined two implicit stimulus classes consisting of B1 and C1 (preferred) and B2 and C2 (nonpreferred), respectively (Dymond \& Rehfeldt, 2001; Goldiamond, 1962). The informal, postexperiment reports of some of these participants are consistent with this interpretation. In particular, these participants reported that 
the pairing of their preferred stimulus, B1, with the aversive slides and noise led them to assume that selecting the discriminative stimuli for their other preferred stimulus, $\mathrm{C} 1$, would also eventually be followed by aversive slides. Interestingly, however, these participants did not differ from the other participants in the number of training blocks required to meet the criterion during the subsequent equivalence training and testing phase of the experiment.

At a second level (see Fig. 3), EC with B1 and B2 affected the stimuli in an equivalence relation with negative $\mathrm{B} 1$ and positive $\mathrm{B} 2$ (i.e., $\mathrm{C} 1$ and $\mathrm{C} 2$, respectively). As compared with the CondNoClass and NoTreat conditions, most CondClass participants shifted their preference from $\mathrm{C} 1$-producing to $\mathrm{C} 2$ producing shape during test II, which strongly suggests the impact of equivalence procedures on the observed shift. These results are consistent with previous studies demonstrating the derived transformation of consequential functions (e.g., Hayes et al., 1991; Whelan \& Barnes-Holmes, 2004a, 2004b; Whelan et al., 2006). However, the test phases in the present study presented singular advantages. First, previous studies employed novel antecedent stimuli whose discriminative functions were then established by the programmed consequences (i.e., presentation of the valenced stimuli). By assessing the baseline rate of selection responses to the shapes that would be used in subsequent tests, this is the first study to show the effects of equivalence training and testing on the alteration of discriminative functions of the associated antecedent stimuli.

Second, the consequences of selection responses in our study were presented only during the initial training of the discriminated operants (i.e., keypresses to square, circle, hexagon, and triangle produced $\mathrm{B} 1, \mathrm{~B} 2, \mathrm{C} 1$, and $\mathrm{C} 2$, respectively), but not during the tests. This was intended to isolate the impact of stimulus relation training procedures on the alteration of preferences from other variables that were also present in previous studies, such as the contingent presentation of the consequences across a large number of trials, as in Hayes et al. (1991), and the inclusion of conditioning trials intermixed with test trials, as in Whelan and Barnes-Holmes (2004a, 2004b).

Findings in test II reveal that stimulus pairing procedures (e.g., B1, then unpleasant stimuli) alter preferences for the conditioned stimulus (e.g., B1) and also for other nonconditioned stimuli (e.g., C1) in an equivalence relation with the former. This adds on traditional formulations of function generalization based on physical similarity and replicates previous findings on the benefits of using MTS procedures when higher-order classical conditioning procedures do not produce the transfer of the EC effect (Barnes-Holmes et al., 2000) - for example, when backward instead of forward conditioning is employed (see Hammerl \& Grabitz, 1996).

Critical in this experiment, EC with B1 and B2, along with the stimulus relations CondClass participants learned during the procedure, had an impact at a third level (see Fig. 3). After being presented with a piece of paper equating B1-, B2-, C1-, and $\mathrm{C} 2$-producing shapes to letters $\mathrm{X}, \mathrm{Y}, \mathrm{W}$, and $\mathrm{Z}$, respectively, participants chose first between B1- and B2-producing letters, and then between $\mathrm{C} 1$ - and $\mathrm{C} 2$-producing letters (remember that selection responses to the letters were never followed by the presentation of $\mathrm{B}$ or $\mathrm{C}$ stimuli). When presented with B1-producing and B2-producing letters (i.e., $\mathrm{X}$ and $\mathrm{Y}$ ), most CondClass and CondNoClass participants preferred the letter established as the B2-producing stimulus for most trials. We say that the discriminative properties of B1producing and $\mathrm{B} 2$-producing shapes changed on the basis of the $\mathrm{EC}$ of the consequences they signaled - that is, B1 and B2, respectively. These properties then transferred to $\mathrm{X}$ and $\mathrm{Y}$, which, in the absence of a history of differential reinforcement, acquired discriminative-like properties. Likewise and more relevant, when presented with C1-producing and C2producing letters (i.e., $\mathrm{W}$ and $\mathrm{Z}$ ), most CondClass participants preferred the $\mathrm{C} 2$-producing letter. This finding is particularly relevant because the discriminative properties of $\mathrm{C} 1$ - and $\mathrm{C} 2-$ producing shapes changed only on the basis of the equivalence established between the consequences they signaled (i.e., $\mathrm{C} 1$ and $\mathrm{C} 2$ ) and directly conditioned $\mathrm{B} 1$ and $\mathrm{B} 2$. The present findings show that discriminative properties altered in such an indirect way may also transfer to novel nonconditioned stimuli. As is shown in Fig. 3, no matter how distant a particular stimulus (e.g., W) is from the event whose valence was altered due to EC (e.g., B1), as long as the individual establishes a connection between them, the preference for the distant stimulus will change as well.

Given the nature of the intervention prior to test III, in which the letters and the shapes appeared together on the same card, it could be argued that the letters acquired discriminative-like functions by stimulus compounding or by any other direct process. However, the transfer of the discriminative functions to the novel stimuli occurred only in the direction of the relations established through the sign " $=$," standing for "equals" or "same as" in our verbal community. Although transfer effects are isolated, further research employing conventional equivalence training procedures is needed, nonetheless, to better isolate the acquisition of discriminative-like properties as observed in test III.

The procedure employed did not allow us to ascertain the independent contribution of the individual stimuli (positive vs. negative) to the observed preference pattern. In line with this, future research may condition a sole function to explore the transformation of preferences for different sets of stimuli according to relations other than equivalence, such as opposition or comparison (e.g., Whelan et al., 2006). This would yield a more solid demonstration of how relational processes may impact the generalized alteration of preferences, in a sort of domino effect.

As is usually recommended in highly controlled laboratory studies, caution should be taken before generalizing these 
findings. Participants underwent the procedures in a distraction-free room and were tested for conditioning and transfer effects immediately after conditioning procedures, equivalence training and testing, and domino effect intervention. It remains to be tested whether these effects can also be observed in uncontrolled settings where equivalence formation and EC may occur in an ongoing manner. For instance, in our procedure, equivalence training and testing occurred once the stimuli had acquired positive and negative valence. Other studies first establish equivalence classes and then use stimulus pairings to alter the valence of stimuli and observe transfer effects (e.g., Rodríguez-Valverde et al., 2009). Although the order seems to be irrelevant, there is some evidence that it takes longer to form equivalence classes when stimuli have emotionally aversive functions, as compared with neutral functions, an effect for which there is no satisfactory explanation to date (Tyndall, Roche, \& James, 2009).

To sum up, using well-known preparations as the departing point, we have (1) isolated the impact of relational training and testing on the derived transformation of consequential functions and (2) come to the first laboratory model of the role of derived relations or relational processes in the generalized alteration of preferences after EC by establishing both within- and between-participants comparisons of the effects. Results showed that after EC (pleasant vs. unpleasant) and equivalence procedures, the behavioral repertoire of experimental, as compared with control, participants became more and more restricted and stereotyped. Further research might elucidate whether this is a useful laboratory model of the generalized alteration of liking across life domains and behavioral narrowing typically observed in some forms of psychopathology, such as PTSD.

Author note Sonsoles Valdivia-Salas is now assistant professor at University of Zaragoza.

This study is part of Sonsoles Valdivia-Salas's doctoral thesis, conducted under the supervision of Carmen Luciano and Michael J. Dougher and funded by a Fulbright fellowship awarded to the first author. Part of this material was presented at the annual convention of the Association for Behavior Analysis, Chicago, May 2005, and Atlanta, May 2006. The authors thank Lanny Fields, William Mcllvane, and two anonymous reviewers for their helpful comments on earlier drafts of the manuscript and Sebastián Lombas for his assistance during data analysis.

Correspondence concerning this article should be addressed to Sonsoles Valdivia-Salas at Departamento de Psicología y Sociología, Universidad de Zaragoza. Campus Ciudad Escolar, 44003 Teruel, Spain.e-mail: sonsoval@unizar.es

\section{References}

Barnes-Holmes, D., Keane, J., Barnes-Holmes, Y., \& Smeets, P. (2000). A derived transfer of emotive functions as a means of establishing differential preferences for soft drinks. The Psychological Record, 50, 493-511.
Beck, A. T., Brown, G. K., \& Steer, R. A. (1996). Beck Depression Inventory-II (BDI-II). San Antonio, TX: The Psychological Corporation.

Carrigan, P. F., \& Sidman, M. (1992). Conditional discrimination and equivalence relations: A theoretical analysis of control by negative stimuli. Journal of the Experimental Analysis of Behavior, 58, 183-204.

Catania, A. C. (1998). Learning. Upper Saddle River, NJ: Prentice Hall.

Center for the Study of Emotion and Attention [CSEA-NIMH]. (2001). The International Affective Picture System: Digitized photographs. Gainesville, FL: The Center for Research in Psychophysiology, University of Florida.

Cicero, S. D., \& Tryon, W. W. (1989). Classical conditioning of meaning - II. A replication and triplet associative extension. Journal of Behavior Therapy and Experimental Psychiatry, 20, 197-202.

Cohen, J. (1988). Statistical power analysis for the behavioral science (2nd ed.). Hillsdale, NJ: Erlbaum.

Colwill, R. M., \& Rescorla, R. A. (1985). Postconditioning devaluation of a reinforcer affects instrumental responding. Journal of Experimental Psychology: Animal Behavior Processes, 11, $120-132$.

Dack, C., McHugh, L., \& Reed, P. (2009). Generalization of causal efficacy judgements after evaluative learning. Learning and Behavior, 37, 336-348.

Dack, C., Reed, P., \& McHugh, L. (2010). Multiple determinants of transfer of evaluative function after conditioning with free-operant schedules of reinforcement. Learning and Behavior, 38, 348-366.

De Houwer, J. (2007). A conceptual and theoretical analysis of evaluative conditioning. The Spanish Journal of Psychology, 10, 230-241.

De Houwer, J., Thomas, S., \& Baeyens, F. (2001). Associative learning of likes and dislikes: A review of 25 years of research on human evaluative conditioning. Psychological Bulletin, 127, 853-869.

Dougher, M. J. (1998). Stimulus equivalence and the untrained acquisition of stimulus functions. Behavior Therapy, 29, 577-591.

Dougher, M. J., Hamilton, D., Fink, B., \& Harrington, J. (2007). Transformation of the discriminative and eliciting functions of generalized relational stimuli. Journal of the Experimental Analysis of Behavior, 88, 179-197.

Dymond, S., \& Rehfeldt, R. A. (2000). Understanding complex behavior: The transformation of stimulus functions. The Behavior Analyst, 23, 239-254.

Dymond, S., \& Rehfeldt, R. A. (2001). Supplemental measures of derived stimulus relations. Experimental Analysis of Human Behavior Bulletin, 19, 8-12.

Gil, E., Luciano, C., Ruiz, F. J., \& Valdivia-Salas, S. (2012). A Preliminary demonstration of transformation of functions through hierarchical relations. International Journal of Psychology and Psychological Therapy, 12, 1-19.

Goldiamond, I. (1962). Perception. In A. J. Bachrach (Ed.), Experimental foundations of clinical psychology (pp. 280-340). New York: Basic Books.

Hammerl, M., \& Grabitz, H. J. (1996). Human evaluative conditioning without experiencing a valued event. Learning and Motivation, 27, 278-293.

Hayes, S. C., Kohlenberg, B. S., \& Hayes, L. J. (1991). The transfer of specific and general consequential functions through simple and conditional equivalence relations. Journal of the Experimental Analysis of Behavior, 56, 119-137.

Hobbs, R. J. (1990). Noise and vibration. In J. Ridley (Ed.), Safety at work. London: Butterworth-Heinemann.

Hoffman, W., De Houwer, J., Perugini, M., Baeyens, F., \& Crombez, G. (2010). Evaluative conditioning in humans: A meta-analysis. Psychological Bulletin, 136, 390-421. 
Hooper, N., Saunders, S., \& McHugh, L. (2010). The derived generalization of thought suppression. Learning and Behavior, 38, $160-168$.

Lang, P. J., Bradley, M. M., \& Cuthbert, B. N. (2001). International Affective Picture System (IAPS): Instructions manual and affective ratings. Technical report A-5. Gainesvillle, FL: The Center for Research in Psychophysiology, University of Florida.

Rodríguez-Valverde, M., Luciano, C., \& Barnes-Holmes, D. (2009). Transfer of aversive respondent elicitation in accordance with equivalence relations. Journal of the Experimental Analysis of Behavior, 92, 85-111.

Sidman, M. (1994). Equivalence relations and behavior: A research story. Boston: Authors Cooperative.

Smeets, P., \& Barnes-Holmes, D. (2003). Children's emergent preferences for soft drinks: Stimulus equivalence and transfer. Journal of Economic Psychology, 24, 603-618.

Smith, M. Y., DuHamel, K., Vickberg, S. J., Papadopoulos, E., Redd, W. H., \& Ricketts, P. (1997). Reliability and validity of the PostTraumatic Stress Disorder Checklist-Civilian (PCL-C). Psychosomatic Medicine, 59, 85.

Smyth, S., Barnes-Holmes, D., \& Forsyth, J. P. (2007). A derived transfer of simple discrimination and self-reported arousal functions in spider fearful and non-spider fearful participants. Journal of the Experimental Analysis of Behavior, 85, 223-246.
Staats, A. W., \& Staats, C. K. (1957). Meaning established by classical conditioning. Journal of Experimental Psychology, 54, 74-80.

Tryon, W. W., \& Cicero, S. D. (1989). Classical conditioning of meaning - I: A replication and higher-order extension. Journal of Behavior Therapy and Experimental Psychiatry, 20, 137-142.

Tyndall, I. T., Roche, B., \& James, J. E. (2009). The interfering effect of emotional stimulus functions on stimulus equivalence class formation: Implications for the understanding and treatment of anxiety. European Journal of Behavior Analysis, 10, 121-140.

Walther, E. (2002). Guilty by mere association: Evaluative conditioning and the spreading attitude effect. Journal of Personality and Social Psychology, 82, 919-934.

Whelan, R., \& Barnes-Holmes, D. (2004a). Empirical models of formative augmenting in accordance with the relations of same, opposite, more-than and less-than. International Journal of Psychology and Psychological Therapy, 4, 285-302.

Whelan, R., \& Barnes-Holmes, D. (2004b). The transformation of consequential functions in accordance with the relational frames of same and opposite. Journal of the Experimental Analysis of Behavior, 82, 177-195.

Whelan, R., Barnes-Holmes, D., \& Dymond, S. (2006). Transfer of the consequential functions in accordance with the relational frames of more-than and less-than. Journal of the Experimental Analysis of Behavior, 86, 317-335. 